\title{
The Influence of Wounding on Nuclear Characteristics of Onion Epidermis
}

\author{
F. B. Kulfinski, R. A. Katsanos, and A. J. Pappelis \\ Department of Biological Sciences, Southern Illinois University, Edwardsville 62025; \\ Department of Biological Sciences, Queensborough Community College, \\ Bayside, New York 11364; and Department of Botany, \\ Southern Illinois University, Carbondale \\ 62901 , U.S.A. respectively
}

Received December 16, 1976

Kulfinski and Pappelis (1975) demonstrated that cells at the cut margin of inner onion epidermis reacted to the injury resulting from cutting by declining in nuclear area (NA) by $59 \%$, increasing in dry mass per unit area of the nucleus (DM/A) by $33 \%$, and decreasing in nuclear dry mass (NDM) by $48 \%$. Similar changes were observed in response to fungal infection (Pappelis and Kulfinski 1971), to fungal products (Kulfinski and Pappelis 1976), and to histological fixation (Kulfinski and Kao, unpublished data). Since such marked changes can be caused to develop in as little as $15 \mathrm{~min}$, we attempted, in this study, to determine the degree and distribution of the trauma resulting from excision of tissue. Our hypothesis was that we would find substantially lower NA and NDM measurements in a marginal band of cells than in the normal cells near the center of the tissue.

\section{Materials and methods}

Inner epidermal tissue squares ( $5 \mathrm{~mm}$ on a side) were obtained from the equatorial region of the third turgid bulb scale of each white onion (Allium cepa L.). NA was determined by photographing nuclei, projecting and tracing their images and measuring these by means of a planimeter. DM/A was calculated from measurements of light retardation with a Leitz interference microscope (Beneke 1966). NDM was obtained as the product of NA $\times$ DM/A (Kulfinski and Pappelis 1976).

In experiment 1 , every third cell (numbers $3,6,9,12,15$, and 18) was measured along straight lines from the margin of the square to the center, exclusive of the first and second cells. The edgemost marginal cells (numbers 1 and 2) were not studied here because their characteristics had previously been documented (Kulfinski and Pappelis 1975). Nine replicates were employed.

In experiment 2, groups of 6 adjacent cells were measured in tissue samples comparable to those previously deseribed. Each marginal group was located among the third through the fifth cell row from the margin and each control group was located near the center of the tissue square. Seven replicates were employed.

\section{Results and discussion}

The influence of wounding on cells adjacent to the cut (cells 1 and 2) was des- 
cribed in a previous study (Kulfinski and Pappelis 1975) which indicated a mean decrease in NDM of $48 \%$ within $15 \mathrm{~min}$ of wounding. Table 1 herein indicates mean values for pooled samples from given locations, 3 being near the cut edge and 18 being near the center of the tissue square. No trends or major differences are evident in any of the nuclear measurements and no statistical analysis was therefore considered necessary.

Table 1. Nuclear characteristics of onion epidermis in relation to distance

from the wounded edge of the tissue. Every third cell was sampled,

3 being the cell closest to the margin and 18 being the closest

to the center of the tissue. Each number is the mean of nine replicates

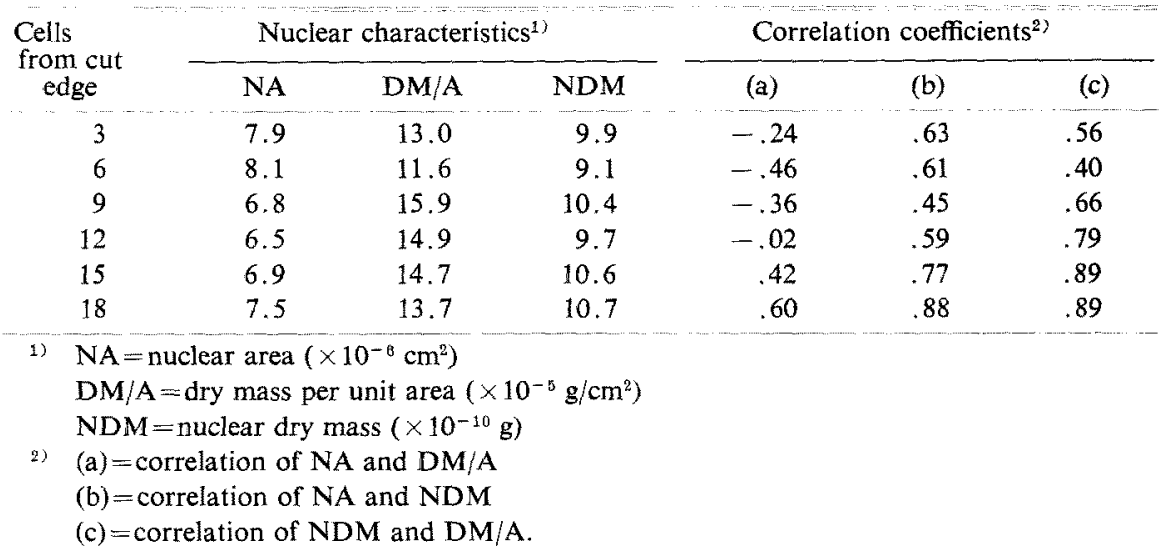

Table 2. Nuclear characteristics of groups of cells in the center and near the wounded margin of a square of epidermal tissue, $5 \mathrm{~mm}$ on a side.

Each number is the mean of 42 nuclei (six nuclei in a group times seven replicates)

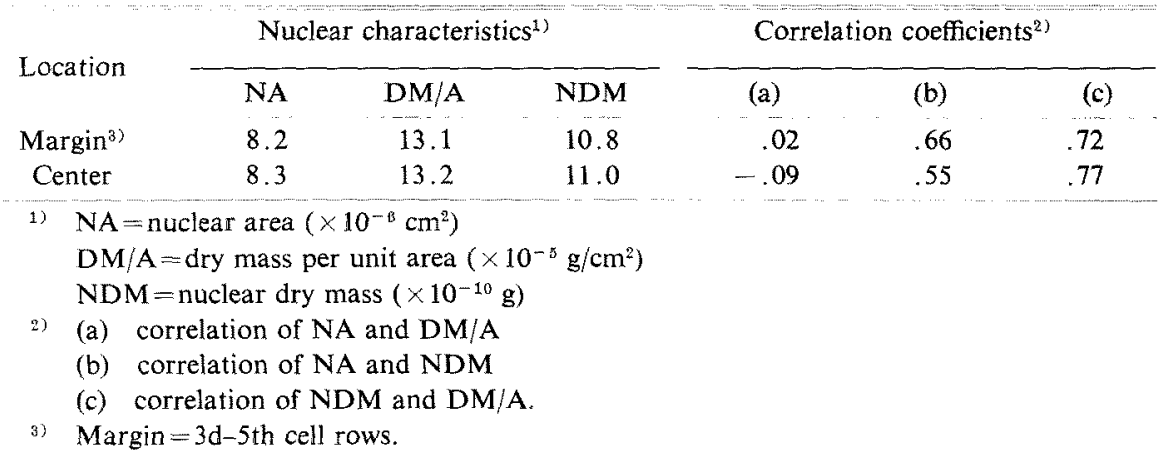

We had hypothesized that cell 3 would be lower in NA and in NDM than the other cell locations (6-18). The NDM of cell 3 was, in fact, greater than that of cell number 6 in absolute quantity and the variation in NDM among all cell locations was sufficient to preclude recognition of any differences.

Table 2 indicates that groups of cells near the margin (cells 3-5) and groups 
near the centers of tissue squares had practically no differences in NA, DM/A, and NDM. We therefore reject our hypothesis that marginal cells in general are lower in NA and NDM than central cells.

Correlation coefficients ( $r$ ) were low and mostly negative between NA and DM/A, ranged from .45 to .88 between NA and NDM, and from .40 to .89 between NDM and DM/A (Tables 1 and 2).

Our general conclusions are that cells at the cut margin decline in nuclear traits rather quickly in response to cutting (Kulfinski and Pappelis 1975), but that practically no change is evident in those cells which are located three or more cell rows in from the margin. It follows that live onion inner epidermal tissue can be used in interferometric studies with little concern as to which cells are used, so long as pycnotic nuclei, mostly in the first two marginal cell row, are recognized and rejected.

\section{Abstract}

Although interference microscopy indicates that onion epidermal cells adjacent to cut margins decline in NDM (nuclear dry mass) by an average of $48 \%$, the present study indicates no differences among nuclei beyond the nucleus of the second cell. Therefore, only nuclei of the extreme margin of cut tissue or internal nuclei exhibiting pycnotic symptoms (perhaps due to handling) need be avoided in sampling. This permits a greater proportion of a piece of tissue to be used in experimentation than was formerly considered feasible (Kulfinski and Pappelis 1975).

\section{Literature cited}

Beneke, G. 1966. Applications of interference microscopy to biological material. In Introduction to Quantitative Cytochemistry (G. L. Wied, ed.), pp. 63-92, Academic Press, New York. Kulfinski, F. B. and Chi-ann Kao. 1974. Unpublished data.

- and Pappelis, A. J. 1975. Interferometric analysis of nuclear pycnosis in injured epidermal cells of Allium sepa. Cytologia 40: 569-571.

- and - 1976. The use of quantitative interferometry in the study of epidermal tissue. In Microbiology of Aerial Plant Surfaces (C. H. Dickinson and T. F. Preece, eds.), pp. 637-657, Academic Press, London.

Pappelis, A. J. and Kulfinski, F. B. 1971. Quantitative interferometry of epidermal nuclei in senescing and infected leaves. In Ecology of Leaf Surface Microorganisms (C. H. Dickinson and T. F. Preece, eds.), pp. 353-366, Academic Press, London. 